\title{
Weighted norm inequalities for convolution and Riesz potential
}

\author{
Erlan Nursultanov and Sergey Tikhonov
}

\begin{abstract}
In this paper, we prove analogues of O'Neil's inequalities for the convolution in the weighted Lebesgue spaces. We also establish the weighted two-sided norm inequalities for the potential operator.
\end{abstract}

\section{Introduction}

In this paper, we study the convolution of functions on $\mathbb{R}^{n}$ :

$$
(K * f)(x)=\int_{\mathbb{R}^{n}} K(x-y) f(y) d y, \quad x \in \mathbb{R}^{n} .
$$

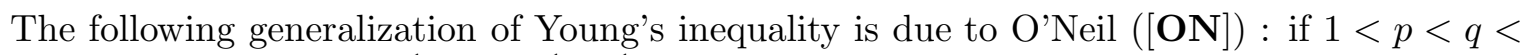
$\infty, \quad 0<\tau_{1} \leqslant \tau_{2} \leqslant \infty$, and $\frac{1}{r}=1-\frac{1}{p}+\frac{1}{q}$, then

$$
L^{p, \tau_{1}} * L^{r, \infty} \subset L^{q, \tau_{2}}
$$

where $L^{p, \tau}=L^{p, \tau}\left(\mathbb{R}^{n}\right)$ is the Lorentz space (see, e.g., [Be, Ch 4]). Throughout the paper the expression of form $X * Y \subset Z$ involving function spaces $X, Y$, and $Z$ means that whenever $f \in X$, $K \in Y$, then $f * K \in Z$ and

$$
\|f * K\|_{Z} \leqslant C\|f\|_{X}\|K\|_{Y}
$$

where the constant $C$ being independent of $f$ and $K$.

A generalization of Young's inequality for the weighted $L^{p}$ spaces was obtained in $[\mathbf{K e}]$. The weighted Lebesgue space $L^{p}(\omega)=L^{p}\left(\omega ; \mathbb{R}^{n}\right)$ consists of all measurable functions such that $\|f\|_{L^{p}(\omega)}=\left(\int_{\mathbb{R}^{n}}|f|^{p} \omega^{p}\right)^{1 / p}<\infty$, where the weight $\omega$ is a nonnegative locally integrable function. Moreover, for the case of power weight $\omega(x)=|x|^{s}$, we write $L^{p}\left(\omega ; \mathbb{R}^{n}\right)=L_{s}^{p}\left(\mathbb{R}^{n}\right)$.

Theorem A. Ke] We have

$$
L_{\alpha}^{p}\left(\mathbb{R}^{n}\right) * L_{s}^{\theta}\left(\mathbb{R}^{n}\right) \subset L_{-\beta}^{q}\left(\mathbb{R}^{n}\right), \quad 1<p, q, \theta<\infty, \quad \frac{1}{q} \leqslant \frac{1}{p}+\frac{1}{\theta},
$$

2000 Mathematics Subject Classification. Primary 31C15; Secondary 44A35, 46E30.

Key words and phrases. Convolution, Riesz potential operator, Weights, Lorentz spaces, O’Neil-type inequalities.

This research was partially supported by the MTM2011-27637/MTM, 2009 SGR 1303, RFFI 12-0100169, NSH 979.2012.1, and Ministry of Education and Science of the Republic of Kazakhstan (0112RK02176, 0112RK00608). 
provided

$$
\begin{gathered}
\frac{1}{q}=\frac{1}{p}+\frac{1}{\theta}+\frac{\alpha+\beta+s}{n}-1, \\
\alpha<n / p^{\prime}, \quad \beta<n / q, \quad s<n / \theta^{\prime},
\end{gathered}
$$

and

$$
\alpha+\beta \geqslant 0, \quad \alpha+s \geqslant 0, \quad \beta+s \geqslant 0 .
$$

Convolution inequalities $L_{\alpha}^{p}\left(\mathbb{R}^{n}\right) * L_{s}^{\theta}\left(\mathbb{R}^{n}\right) \subset L_{-\beta}^{q}\left(\mathbb{R}^{n}\right)$ were also studied in [Bu1]. Conditions (1.3) - (1.5) are necessary as it is shown in Section 7 .

Let us now formulate the first question studied in this paper:

Problem 1. Find sufficient conditions on weights $\mu$ and $\nu$, so that

$$
L^{p}(\mu) * L^{r, \infty} \subset L^{q}(\nu), \quad 1<p \leqslant q<\infty, \quad 1<r \leqslant \infty .
$$

Further, we deal with an important example of the convolution operator $K * f$, where $K(z)=|z|^{\gamma-n}, z \in \mathbb{R}^{n}$, i.e., the operator of the fractional integration (or Riesz potential):

$$
I_{\gamma} f(x)=\int_{\mathbb{R}^{n}} \frac{f(y)}{|x-y|^{n-\gamma}} d y, \quad 0<\gamma<n .
$$

For this operator, (1.1) implies $I_{\gamma}: L^{p, \tau_{1}}\left(\mathbb{R}^{n}\right) \rightarrow L^{q, \tau_{2}}\left(\mathbb{R}^{n}\right)$ for $\frac{1}{p}-\frac{1}{q}=\frac{\gamma}{n}$ and $0<\tau_{1} \leqslant \tau_{2} \leqslant \infty$. In particular, this yields the Hardy-Littlewood-Sobolev theorem, i.e., $I_{\gamma}: L^{p}\left(\mathbb{R}^{n}\right) \rightarrow L^{q}\left(\mathbb{R}^{n}\right)$. Continuity properties of the potential operator in the Lebesgue spaces are well known, see, e.g., [BS], So], [SW].

Analogous questions have been intensively investigated in the weighted Lebesgue spaces. For the power weights, Stein and Weiss [SW1 generalized the Hardy-Littlewood-Sobolev theorem as follows: Suppose

$$
\gamma=\alpha+\beta+n(1 / p-1 / q), \quad \alpha<n / p^{\prime}, \quad \beta<n / q, \quad 0 \leqslant \alpha+\beta,
$$

then

$$
I_{\gamma}: L_{\alpha}^{p}\left(\mathbb{R}^{n}\right) \rightarrow L_{-\beta}^{q}\left(\mathbb{R}^{n}\right), \quad 1<p \leqslant q<\infty .
$$

For arbitrary weights $\mu$ and $\nu$, the solution of the problem stated as "give necessary and sufficient conditions on weights for the Riesz potential $I_{\gamma}$ to be bounded from $L^{p}\left(\mu, \mathbb{R}^{n}\right)$ to $L^{q}\left(\nu, \mathbb{R}^{n}\right)$ ", can

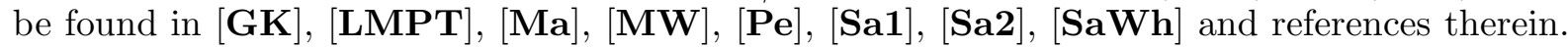
In particular, it is known (see [LMPT, (2.2)]) that for $1<p \leqslant q<\infty$,

$$
\left\|I_{\gamma}\right\|_{L^{p}\left(\mu, \mathbb{R}^{n}\right) \rightarrow L^{q}\left(\nu, \mathbb{R}^{n}\right)} \asymp\left\|I_{\gamma}\right\|_{L^{q^{\prime}}\left(\nu^{1-q^{\prime}}, \mathbb{R}^{n}\right) \rightarrow L^{p^{\prime}, \infty}\left(\mu^{1-p^{\prime}}, \mathbb{R}^{n}\right)}+\left\|I_{\gamma}\right\|_{L^{p}\left(\mu, \mathbb{R}^{n}\right) \rightarrow L^{q, \infty}\left(\nu, \mathbb{R}^{n}\right)} .
$$

Note also that 'upper' triangle case with one weight was considered in [Ma and [MN] in terms of capacity; a non-capacity characterization was proved in [COV].

Our second goal in this paper is to study two-sided norm inequalities for the weighted potential operator

$$
\left(A_{\gamma} f\right)(y)=\left(A_{\gamma, \mu, \nu} f\right)(y)=\nu(y) \int_{\mathbb{R}^{n}} \frac{f(x) \mu(x)}{|x-y|^{n-\gamma}} d x, \quad y \in \mathbb{R}^{n}, \quad 0<\gamma<n,
$$

in the Lorentz spaces. More precisely, we study the following question.

Problem 2. Find upper and lower bounds of the norm of the operator $A_{\gamma}: L^{p, \tau_{1}}\left(\mathbb{R}^{n}\right) \rightarrow$ $L^{q, \tau_{2}}\left(\mathbb{R}^{n}\right), 1<p, q<\infty, 1<\tau_{1}, \tau_{2} \leqslant \infty$, in terms of $\mu$ and $\nu$. 
Note that this question is similar to the following problem: to estimate the norm of the potential operator in the weighted Lorentz space, i.e., $I_{\gamma}: L^{p, \tau_{1}}\left(\mu^{-1}, \mathbb{R}^{n}\right) \rightarrow L^{q, \tau_{2}}\left(\nu, \mathbb{R}^{n}\right)$, which is, in general, a different question. The latter case was thoroughly studied by Kerman for the power weights; see Theorems 4.1 and 4.5 in $[\mathbf{K e}$.

In this paper $F \lesssim G$ means that $F \leqslant C G$; by $C$ we denote positive constants that may be different on different occasions. Moreover, $F \asymp G$ means that $F \lesssim G \lesssim F$.

For a Lebesgue measurable set $E,|E|$ will denote its Lebesgue measure and $\nu(E)=\int_{E} \nu(x) d x$ will denote its weighted measure. If $E$ and $W$ are any subsets of $\mathbb{R}^{n}$, the Minkowski sum of $E$ and $W$ is defined by $E+W=\{x+y: x \in E$ and $y \in W\}$. The characteristic function of a set $E$ is denoted by $\chi_{E}$. Let also $\overline{0}=(0, \ldots, 0), \bar{N}=(N, \ldots, N)$, and $B_{r}(\bar{N})=\left\{x \in \mathbb{R}^{n}:|x-\bar{N}| \leqslant r\right\}$. As usual, $\psi^{*}$ is the decreasing rearrangement of $\psi$ see, e.g., [BS].

Finally, $X^{\prime}$ is the associate space of the space $X$, i.e., $X^{\prime}=\left\{g:\|g\|_{X^{\prime}}=\sup _{\|f\|_{X} \leqslant 1}\left|\int f g\right|<\infty\right\}$.

\section{Main results}

First, we give a solution of Problem 1 by proving the following extension of Theorem A.

TheOREM 1. Let $1<p \leqslant q<\infty$ and $1<r<\infty$. Let weights $\mu$ and $\nu$ satisfy, for any $\lambda \geqslant 1$

$$
\begin{aligned}
\mu^{*}(\lambda t) \lesssim \frac{\mu^{*}(t)}{\lambda^{\alpha}}, & t>0, \\
\nu^{*}(\lambda t) \lesssim \frac{\nu^{*}(t)}{\lambda^{\beta}}, & t>0,
\end{aligned}
$$

for some $\alpha \geqslant 0$ and $\beta \geqslant 0$ such that

$$
\alpha+1 / r+1 / p>1, \quad \beta+1 / r+1 / q^{\prime}>1 .
$$

Then a sufficient condition for

$$
L^{p}\left(\mu^{-1}\right) * L^{r, \infty} \subset L^{q}(\nu)
$$

to hold is

$$
\mathcal{G}:=\sup _{|E|=|W|} \frac{\nu(E) \mu(W)}{|E|^{1+\frac{1}{r}+\frac{1}{p}-\frac{1}{q}}}<\infty,
$$

where the supremum is taken over all measurable sets $E$ and $W$ of the same positive measure. A necessary condition for 2.4) is

$$
\mathcal{S}:=\sup _{|E|=|W|} \frac{\nu(E) \mu(W)}{|E|^{1+\frac{1}{p}-\frac{1}{q}}|E-W|^{\frac{1}{r}}}<\infty,
$$

where the supremum is taken over all measurable sets $E$ and $W$ of the same positive measure.

REMARK. Note that conditions (2.1) and (2.2) are fulfilled for arbitrary weights $\mu$ and $\nu$ with $\alpha=\beta=0$. Hence, the statement of Theorem 1 holds for arbitrary $\mu$ and $\nu$ if $1 / r+1 / p>1$ and $1 / r+1 / q^{\prime}>1$, cf. (2.3). Similar remarks can be applied to Theorem 2 and Corollary 2 below. Let us also note that if a function $\psi$ is $\delta$-regularly varying (see [BGT, Sect. 2]), that is, it satisfies $\psi(\lambda t) \lesssim \frac{\psi(t)}{\lambda^{\delta}}$, then it also satisfies $\psi^{*}(\lambda t) \lesssim \frac{\psi^{*}(t)}{\lambda^{\delta}}$. 
For the power weights $\mu(x)=|x|^{-\alpha}$ and $\nu(x)=|x|^{-\beta}$, Theorem 1 implies necessary and sufficient conditions for (2.4) to hold.

Corollary 1. Suppose $1<p \leqslant q<\infty$ and $1<r<\infty$. Then

$$
L_{\alpha}^{p}\left(\mathbb{R}^{n}\right) * L^{r, \infty}\left(\mathbb{R}^{n}\right) \subset L_{-\beta}^{q}\left(\mathbb{R}^{n}\right)
$$

if and only if

$$
\frac{1}{q}=\frac{1}{p}+\frac{1}{r}+\frac{\alpha+\beta}{n}-1, \quad 0 \leqslant \alpha<n / p^{\prime}, \quad 0 \leqslant \beta<n / q .
$$

In particular, this implies the statement of Theorem $A$ in case when $\alpha, \beta, s \geqslant 0$.

If $\alpha=\beta=0$, (2.7) is O'Neil's inequality $L^{p}\left(\mathbb{R}^{n}\right) * L^{r, \infty}\left(\mathbb{R}^{n}\right) \subset L^{q}\left(\mathbb{R}^{n}\right), \frac{1}{q}=\frac{1}{p}+\frac{1}{r}-1$. Moreover, (2.7) gives the Stein-Weiss inequality (1.8) under conditions (1.7) and $\alpha, \beta \geqslant 0$.

Let us now study Problem 2. The following result gives upper and lower norm estimates of the weighted potential operator $A_{\gamma}$ in the Lorentz spaces.

Theorem 2. Let $1<p, q<\infty, 0<\tau_{1} \leqslant \tau_{2} \leqslant \infty$, and $0<\gamma<n$.

(A). Let the weights $\mu$ and $\nu$ satisfy conditions (2.1) and (2.2) for some $\alpha \geqslant 0$ and $\beta \geqslant 0$ such that

$$
\alpha+1 / p>\gamma / n, \quad \beta+1 / q^{\prime}>\gamma / n
$$

If

$$
\mathcal{L}:=\sup _{|E|=|W|} \frac{\nu(E) \mu(W)}{|E|^{2+\frac{1}{p}-\frac{1}{q}-\frac{\gamma}{n}}}<\infty
$$

where the supremum is taken over all measurable sets $E$ and $W$ of the same positive measure, then $A_{\gamma}$ is bounded from $L^{p, \tau_{1}}\left(\mathbb{R}^{n}\right)$ to $L^{q, \tau_{2}}\left(\mathbb{R}^{n}\right)$ and

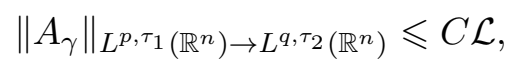

where $C$ depends only on parameters $p, q, \tau_{1}, \tau_{2}, \alpha, \beta, \gamma$, and $n$.

(B). If the operator $A_{\gamma}$ is bounded from $L^{p, \tau_{1}}\left(\mathbb{R}^{n}\right)$ to $L^{q, \tau_{2}}\left(\mathbb{R}^{n}\right)$, then

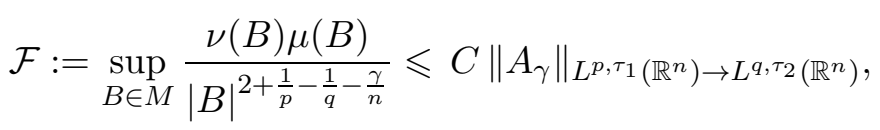

where $M$ is the collection of all balls in $\mathbb{R}^{n}$ and $C$ depends only on parameters $p, q, \tau_{1}, \tau_{2}, \alpha, \beta$, $\gamma$, and $n$.

Remark. Comparing $\mathcal{S}$ and $\mathcal{F}$ defined by (2.6) and (2.11), we see that

$$
\mathcal{F} \leqslant \sup _{x \in \mathbb{R}^{n}, E \text { convex }} \frac{\nu(E) \mu(E+x)}{|E|^{1+\frac{1}{r}+\frac{1}{p}-\frac{1}{q}}} \lesssim \mathcal{S} .
$$

Taking $\tau_{1}=p$ and $\tau_{2}=q$ in Theorem 2 , we get $\mathcal{F} \lesssim\left\|A_{\gamma}\right\|_{L^{p}\left(\mathbb{R}^{n}\right) \rightarrow L^{q}\left(\mathbb{R}^{n}\right)} \lesssim \mathcal{L}$. Moreover, under certain regularity conditions on weights, the left-hand side bound $\mathcal{F}$ and the right-hand side bound $\mathcal{L}$ are equivalent. In particular, this holds for radial weights $\psi(|x|)$ satisfying the following condition:

$$
\psi^{*}(t) \lesssim \frac{1}{t} \int_{t / 2}^{t} \psi(s) d s, \quad t>0
$$


Note that (2.12) holds for any monotonic function $\psi$ on $(0, \infty)$. It is easy to give an example of a non-monotonic function satisfying condition (2.12), for example,

$$
\psi(t)=\frac{|\cos n t|}{t^{\alpha}}, \quad t>0, \quad 0<\alpha<1 .
$$

Theorem 2 implies the following relation for the norm of the weighted potential operator.

Corollary 2. Let $p, q, \gamma, \tau_{1}, \tau_{2}$ satisfy all conditions of Theorem Q Suppose functions $\nu_{0}(\cdot)$ and $\mu_{0}(\cdot)$ defined on $(0, \infty)$ satisfy condition (2.12). Then, for the operator

$$
\left(A_{\gamma} f\right)(y)=\left(A_{\gamma, \mu, \nu} f\right)(y)=\nu_{0}(|y|) \int_{\mathbb{R}^{n}} \frac{f(x) \mu_{0}(|x|)}{|x-y|^{n-\gamma}} d x, \quad y \in \mathbb{R}^{n}, \quad 0<\gamma<n,
$$

one has

$$
\left\|A_{\gamma}\right\|_{L^{p, \tau_{1}}\left(\mathbb{R}^{n}\right) \rightarrow L^{q, \tau_{2}}\left(\mathbb{R}^{n}\right)} \asymp \sup _{a>0} \frac{1}{a^{n\left(2+\frac{1}{p}-\frac{1}{q}-\frac{\gamma}{n}\right)}} \int_{0}^{a} \nu_{0}(r) r^{n-1} d r \int_{0}^{a} \mu_{0}(r) r^{n-1} d r .
$$

In particular, if $1<p \leqslant q<\infty$, then

$$
\left\|A_{\gamma}\right\|_{L^{p}\left(\mathbb{R}^{n}\right) \rightarrow L^{q}\left(\mathbb{R}^{n}\right)} \asymp \sup _{a>0} \frac{1}{a^{n\left(2+\frac{1}{p}-\frac{1}{q}-\frac{\gamma}{n}\right)}} \int_{0}^{a} \nu_{0}(r) r^{n-1} d r \int_{0}^{a} \mu_{0}(r) r^{n-1} d r .
$$

In the case of the power weights $\mu(x)=|x|^{-\alpha}$ and $\nu(x)=|x|^{-\beta}$ we obtain necessary and sufficient conditions for boundedness of the operator $A_{\gamma}$.

Corollary 3. Let $1<p, q<\infty, \alpha \geqslant 0$, and $\beta \geqslant 0$.

(A). Let either $0<\tau_{1} \leqslant \tau_{2}<\infty$ or $1<\tau_{1} \leqslant \tau_{2} \leqslant \infty$. Then the operator

$$
\left(A_{\gamma, \alpha, \beta} f\right)(y)=\int_{\mathbb{R}^{n}} \frac{f(x)}{|x|^{\alpha}|x-y|^{n-\gamma}|y|^{\beta}} d x, \quad y \in \mathbb{R}^{n}, \quad 0<\gamma<n,
$$

is bounded from $L^{p, \tau_{1}}\left(\mathbb{R}^{n}\right)$ to $L^{q, \tau_{2}}\left(\mathbb{R}^{n}\right)$, if and only if

$$
\gamma=\alpha+\beta+n\left(\frac{1}{p}-\frac{1}{q}\right), \quad \alpha<\frac{n}{p^{\prime}}, \quad \beta<\frac{n}{q} .
$$

(B). Let $0<\tau \leqslant 1$. The operator $A_{\gamma, \alpha, \beta}$ is bounded from $L^{p, \tau}\left(\mathbb{R}^{n}\right)$ to $L^{q, \infty}\left(\mathbb{R}^{n}\right)$ if and only if

$$
\gamma=\alpha+\beta+n\left(\frac{1}{p}-\frac{1}{q}\right), \quad \alpha \leqslant \frac{n}{p^{\prime}}, \quad \beta \leqslant \frac{n}{q} .
$$

In particular, for the Lebesgue spaces this result is reduced to $I_{\gamma}: L_{\alpha}^{p}\left(\mathbb{R}^{n}\right) \rightarrow L_{-\beta}^{q}\left(\mathbb{R}^{n}\right)$, $1<p \leqslant q<\infty$, that is, (1.8). Note that necessity of conditions (1.7) was discussed in [Du].

\section{Lemmas}

Define

$$
f^{* *}(t)=\frac{1}{t} \int_{0}^{t} f^{*}(s) d s
$$


We will use the fact (see [BS, p. 53]) that $f^{* *}$ can be written as follows:

$$
f^{* *}(t)=\sup _{|E|=t} \frac{1}{|E|} \int_{E}|f(x)| d x
$$

Lemma 1. Let $W$ and $E$ be measurable sets in $\mathbb{R}^{n}$, then

$$
\left|\int_{W} \mu(x) \int_{E} \nu(y) K(y-x) d y d x\right| \leqslant \int_{0}^{|W|} \mu^{*}(s) \int_{0}^{|E|} \nu^{*}(t) K^{* *}(\max (t, s)) d t d s .
$$

Proof. The proof is inspired by ideas from the paper [NT1]. By the Hardy-Littlewood rearrangement inequality

$$
\begin{aligned}
\int_{W} \mu(x) \int_{E} \nu(y) K(y-x) d y d x & \leqslant \int_{0}^{|W|} \mu^{*}(s)\left(\int_{E} \nu(y) K(y-\cdot) d y\right)^{*}(s) d s \\
& \leqslant \int_{0}^{|W|} \mu^{*}(s)\left(\int_{E} \nu(y) K(y-\cdot) d y\right)^{* *}(s) d s \\
& =\int_{0}^{|W|} \mu^{*}(s) \sup _{\left|\eta_{1}\right|=s} \frac{1}{\left|\eta_{1}\right|} \int_{\eta_{1}}\left|\int_{E} \nu(y) K(y-x) d y\right| d x d s \\
& \leqslant \int_{0}^{|W|} \mu^{*}(s) \sup _{\left|\eta_{1}\right|=s}\left(\frac{1}{\left|\eta_{1}\right|} \int_{E}|\nu(y)| \int_{\eta_{1}}|K(y-x)| d x d y\right) d s
\end{aligned}
$$

We use similar estimates for the inner integral to get

$$
\begin{aligned}
\int_{W} \mu(x) \int_{E} \nu(y) K(y-x) d y d x & \leqslant \int_{0}^{|W|} \mu^{*}(s) \sup _{\left|\eta_{1}\right|=s} \int_{0}^{|E|} \nu^{*}(t) \sup _{\left|\eta_{2}\right|=t} \frac{1}{\left|\eta_{2}\right|} \frac{1}{\left|\eta_{1}\right|} \int_{\eta_{2}} \int_{\eta_{1}}|K(y-x)| d x d y d t d s \\
& \leqslant \int_{0}^{|W|} \mu^{*}(s) \int_{0}^{|E|} \nu^{*}(t) \sup _{\left|\eta_{1}\right|=s\left|\eta_{2}\right|=t} \sup _{\left|\eta_{2}\right|} \frac{1}{\left|\eta_{1}\right|} \int_{\eta_{2}} \int_{\eta_{1}}|K(y-x)| d x d y d t d s
\end{aligned}
$$

If $t \geqslant s$, then

$$
\begin{aligned}
& \sup _{\left|\eta_{1}\right|=s} \sup _{\left|\eta_{2}\right|=t} \frac{1}{\left|\eta_{2}\right|} \frac{1}{\left|\eta_{1}\right|} \int_{\eta_{2}} \int_{\eta_{1}}|K(y-x)| d x d y \\
& \leqslant \sup _{\left|\eta_{1}\right|=s} \frac{1}{\left|\eta_{1}\right|} \int_{\eta_{1}} \sup _{\left|\eta_{2}\right|=t} \frac{1}{\left|\eta_{2}\right|} \int_{\eta_{2}}|K(y-x)| d y d x=K^{* *}(t) .
\end{aligned}
$$

Similarly, if $t<s$, then

$$
\sup _{\left|\eta_{1}\right|=s\left|\eta_{2}\right|=t} \sup _{\left|\eta_{2}\right|} \frac{1}{\left|\eta_{1}\right|} \int_{\eta_{2}} \int_{\eta_{1}}|K(y-x)| d x d y \leqslant K^{* *}(s)
$$

and the statement follows.

\section{Lemma 2. Let $\beta>1$. Suppose}

$$
\mathcal{B}=\sup _{\omega>0} \frac{1}{\omega^{\beta}} \int_{0}^{\omega} \mu^{*}(s) d s \int_{0}^{\omega} \nu^{*}(t) d t \int_{0}^{\omega} K^{*}(s) d s<\infty .
$$

Then there exists $C$ depending only on $\beta$ such that

$$
\sup _{\omega>0} \frac{1}{\omega^{\beta-1}} \int_{0}^{\omega} \mu^{*}(s) \int_{0}^{\omega} \nu^{*}(t) K^{* *}(\max (t, s)) d t d s \leqslant C \mathcal{B} .
$$


Proof. For $\omega>0$ we have

$$
\begin{aligned}
& \frac{1}{\omega^{\beta-1}} \int_{0}^{\omega} \mu^{*}(s) \int_{0}^{\omega} \nu^{*}(t) K^{* *}(\max (t, s)) d t d s \\
& =\frac{1}{\omega^{\beta-1}} \int_{0}^{\omega} \mu^{*}(s)\left(\int_{0}^{s} \nu^{*}(t) K^{* *}(s) d t+\int_{s}^{\omega} \nu^{*}(t) K^{* *}(t) d t\right) d s \\
& =\frac{1}{\omega^{\beta-1}} \int_{0}^{\omega} K^{* *}(s)\left(\int_{0}^{s} \nu^{*}(t) d t \int_{0}^{s} \mu^{*}(t) d t\right)^{\prime} d s \\
& =\frac{1}{\omega^{\beta-1}}\left[\left.K^{* *}(s) \int_{0}^{s} \nu^{*}(t) d t \int_{0}^{s} \mu^{*}(t) d t\right|_{0} ^{\omega}-\int_{0}^{\omega}\left(K^{* *}(s)\right)^{\prime} \int_{0}^{s} \nu^{*}(t) d t \int_{0}^{s} \mu^{*}(t) d t d s\right] \\
& =: I_{1}+I_{2} .
\end{aligned}
$$

To estimate $I_{2}$, taking into account that

$$
-\left(K^{* *}(s)\right)^{\prime}=\frac{1}{s}\left(K^{* *}(s)-K^{*}(s)\right) \leqslant \frac{1}{s} K^{* *}(s),
$$

we get

$$
I_{2} \leqslant \frac{1}{\omega^{\beta-1}} \int_{0}^{\omega} K^{* *}(s)\left(\int_{0}^{s} \nu^{*}(t) d t \int_{0}^{s} \mu^{*}(t) d t\right) \frac{d s}{s} .
$$

By (3.2)

$$
I_{2} \leqslant \frac{\mathcal{B}}{\omega^{\beta-1}} \int_{0}^{\omega} \frac{d s}{s^{2-\beta}} \lesssim \mathcal{B} .
$$

Let us estimate $I_{1}$. We have

$$
\begin{aligned}
I_{1} & =\frac{1}{\omega^{\beta}} \int_{0}^{\omega} K^{*}(t) d t \int_{0}^{\omega} \nu^{*}(t) d t \int_{0}^{\omega} \mu^{*}(t) d t-\frac{1}{\omega^{\beta-1}} \lim _{s \rightarrow 0+} \frac{1}{s} \int_{0}^{s} K^{*}(t) d t \int_{0}^{s} \nu^{*}(t) d t \int_{0}^{s} \mu^{*}(t) d t \\
& \leqslant \mathcal{B}+\frac{1}{\omega^{\beta-1}}\left|\lim _{s \rightarrow 0+} \frac{1}{s} \int_{0}^{s} K^{*}(t) d t \int_{0}^{s} \nu^{*}(t) d t \int_{0}^{s} \mu^{*}(t) d t\right| .
\end{aligned}
$$

Since

$$
\frac{1}{s} \int_{0}^{s} K^{*}(t) d t \int_{0}^{s} \nu^{*}(t) d t \int_{0}^{s} \mu^{*}(t) d t \leqslant s^{\beta-1} \mathcal{B}, \quad s>0,
$$

then

$$
\lim _{s \rightarrow 0+} \frac{1}{s} \int_{0}^{s} K^{*}(t) d t \int_{0}^{s} \nu^{*}(t) d t \int_{0}^{s} \mu^{*}(t) d t=0
$$

and therefore, $I_{1} \leqslant \mathcal{B}$.

Lemma 3. Let $1<p, q<\infty$. Let $\mu, \nu$, and $K$ be locally integrable functions on $\mathbb{R}^{n}$ and satisfy, for any $\lambda \geqslant 1$,

$$
\mu^{*}(\lambda t) \lesssim \frac{\mu^{*}(t)}{\lambda^{\alpha}}, \quad \nu^{*}(\lambda t) \lesssim \frac{\nu^{*}(t)}{\lambda^{\beta}}, \quad K^{*}(\lambda t) \lesssim \frac{K^{*}(t)}{\lambda^{\rho}}, \quad t>0,
$$

for some $\alpha \geqslant 0, \beta \geqslant 0$, and $\rho \geqslant 0$. Let also

$$
\rho+\alpha+1 / p \geqslant 1, \quad \rho+\beta+1 / q^{\prime} \geqslant 1 .
$$

If

$$
\mathcal{D}:=\sup _{t>0} \frac{1}{t^{2+1 / p-1 / q}} \int_{0}^{t} \mu^{*}(s) d s \int_{0}^{t} \nu^{*}(s) d s \int_{0}^{t} K^{*}(s) d s<\infty,
$$


then

$$
\sup _{\substack{\xi>0 \\ \eta>0}} \frac{1}{\eta^{\frac{1}{q^{\prime}}}} \frac{1}{\xi^{\frac{1}{p}}} \int_{0}^{\eta} \nu^{*}(t) \int_{0}^{\xi} \mu^{*}(s) K^{* *}(\max (s, t)) d s d t \leqslant C \mathcal{D},
$$

where $C$ depends only on $p, q, \alpha, \beta, \rho$.

Proof. Suppose also that $\eta>\xi>0$. We take an integer $N>1$ such that $(N-1) \xi<\eta \leqslant$ $N \xi$. Then

$$
\begin{aligned}
& \frac{1}{\eta^{\frac{1}{q^{\prime}}}} \frac{1}{\xi^{\frac{1}{p}}} \int_{0}^{\eta} \nu^{*}(t) \int_{0}^{\xi} \mu^{*}(s) K^{* *}(\max (s, t)) d s d t \\
& \leqslant \frac{1}{((N-1) \xi)^{\frac{1}{q^{\prime}}}} \frac{1}{\xi^{\frac{1}{p}}} \int_{0}^{\eta} \nu^{*}(t) \int_{0}^{\xi} \mu^{*}(s) K^{* *}(\max (s, t)) d s d t \\
& \lesssim \frac{1}{((N-1) \xi)^{1 / q^{\prime}} \xi^{1 / p}} \int_{0}^{\xi} \mu^{*}(s) \sum_{k=1}^{N} \int_{(k-1) \xi}^{k \xi} \nu^{*}(t) K^{* *}(\max (s, t)) d t d s .
\end{aligned}
$$

We divide the last expression into two terms:

$$
\begin{gathered}
\frac{1}{((N-1) \xi)^{1 / q^{\prime}} \xi^{1 / p}} \int_{0}^{\xi} \mu^{*}(s) \int_{0}^{\xi} \nu^{*}(t) K^{* *}(\max (s, t)) d t d s \\
+\frac{1}{((N-1) \xi)^{1 / q^{\prime}} \xi^{1 / p}} \int_{0}^{\xi} \mu^{*}(s) \sum_{k=2}^{N} \int_{(k-1) \xi}^{k \xi} \nu^{*}(t) K^{* *}(t) d t d s=: J_{1}+J_{2} .
\end{gathered}
$$

Note that (3.4) implies

$$
K^{* *}(\lambda t) \lesssim \frac{K^{* *}(t)}{\lambda^{\rho}}
$$

Indeed,

$$
\begin{aligned}
K^{* *}(\lambda t)=\frac{1}{\lambda t} \int_{0}^{\lambda t} K^{*}(s) d s & =\frac{1}{t} \int_{0}^{t} K^{*}(\lambda s) d s \\
& \lesssim \frac{1}{t} \int_{0}^{t} \frac{K^{*}(s)}{\lambda^{\rho}} d s=\frac{K^{* *}(t)}{\lambda^{\rho}} .
\end{aligned}
$$

Therefore, using $\nu^{*}(\lambda t) \lesssim \lambda^{-\beta} \nu^{*}(t)$ and (3.6) $)$, we get

$$
\begin{aligned}
J_{2} & \lesssim \frac{1}{((N-1) \xi)^{1 / q^{\prime}} \xi^{1 / p}} \int_{0}^{\xi} \mu^{*}(s) d s \sum_{k=2}^{N} \nu^{*}((k-1) \xi) K^{* *}((k-1) \xi) \xi \\
& \lesssim \frac{1}{((N-1) \xi)^{1 / q^{\prime}} \xi^{1 / p}} \int_{0}^{\xi} \mu^{*}(s) d s \int_{0}^{\xi} \nu^{*}(t) d t K^{* *}(\xi) \sum_{k=2}^{N} \frac{1}{(k-1)^{\rho+\beta}} .
\end{aligned}
$$

Noting that $\rho+\beta+1 / q^{\prime} \geqslant 1$, we get

$$
J_{2} \lesssim \mathcal{D}
$$

Estimating $J_{1}$, we use Lemma 2 to get $J_{1} \lesssim \mathcal{D}$. 
Summing the estimates for $J_{1}$ and $J_{2}$, we finally have

$$
\frac{1}{\eta^{\frac{1}{q^{\prime}}}} \frac{1}{\xi^{\frac{1}{p}}} \int_{0}^{\eta} \nu^{*}(t) \int_{0}^{\xi} \mu^{*}(s) K^{* *}(\max (s, t)) d s d t \lesssim \mathcal{D}
$$

in the case when $\eta>\xi$.

If $\xi \geqslant \eta$, then we use similar estimates and the condition $\rho+\alpha+1 / p \geqslant 1$.

\section{Proof of Theorem 1}

Proof. We consider the operator

$$
(A f)(y)=\left(A_{K, \mu, \nu} f\right)(y)=\nu(y) \int_{\mathbb{R}^{n}} f(x) K(x-y) \mu(x) d x, \quad y \in \mathbb{R}^{n} .
$$

Let us first prove that the operator $A$ is a $(p, q)$ quasi-weak-type operator, i.e.,

$$
\|A\|_{L^{p, 1}\left(\mathbb{R}^{n}\right) \rightarrow L^{q, \infty}\left(\mathbb{R}^{n}\right)}<C^{*},
$$

where $C^{*}=C(p, q, r) \mathcal{G}\|K\|_{L^{r, \infty}\left(\mathbb{R}^{n}\right)}$. By Corollary 4.1 from the paper [NT2], we have

$$
\|A\|_{L^{p, 1}\left(\mathbb{R}^{n}\right) \rightarrow L^{q, \infty}\left(\mathbb{R}^{n}\right)} \asymp \sup _{\substack{|W|>0 \\|E|>0}} \frac{1}{|E|^{\frac{1}{q^{q}}}} \frac{1}{|W|^{\frac{1}{p}}}\left|\int_{E} \nu(y) \int_{W} K(y-x) \mu(x) d x d y\right|
$$

and hence, it is enough to show that the latter is bounded.

Let $E$ and $W$ be any measurable sets from $\mathbb{R}^{n}$ of positive measure. Lemma 1 yields

$$
\begin{aligned}
& \frac{1}{|E|^{\frac{1}{q^{\prime}}}} \frac{1}{|W|^{\frac{1}{p}}} \int_{E} \nu(y) \int_{W} K(y-x) \mu(x) d x d y \\
& \leqslant \quad \frac{1}{|E|^{\frac{1}{q^{\prime}}}} \frac{1}{|W|^{\frac{1}{p}}} \int_{0}^{|E|} \nu^{*}(t) \int_{0}^{|W|} \mu^{*}(s) K^{* *}(\max (s, t)) d s d t \\
& \leqslant \quad\left(\sup _{t>0} \frac{1}{t^{1-\frac{1}{r}}} \int_{0}^{t} K^{*}(s) d s\right) \frac{1}{|E|^{\frac{1}{q^{\prime}}}} \frac{1}{|W|^{\frac{1}{p}}} \int_{0}^{|E|} \nu^{*}(t) \int_{0}^{|W|} \mu^{*}(s) \frac{1}{\max (s, t)^{\frac{1}{r}}} d s d t \\
& \lesssim \quad\|K\|_{L^{r, \infty}} \frac{1}{|E|^{\frac{1}{q^{\prime}}}} \frac{1}{|W|^{\frac{1}{p}}} \int_{0}^{|E|} \nu^{*}(t) \int_{0}^{|W|} \mu^{*}(s) \frac{1}{\max (s, t)^{\frac{1}{r}}} d s d t .
\end{aligned}
$$

Since the function $\varphi(x)=|x|^{-n / r}$ satisfies $\varphi^{* *}(s) \asymp s^{-1 / r}$, Lemma 3 gives

$$
\begin{aligned}
& \frac{1}{|E|^{\frac{1}{q^{\prime}}}} \frac{1}{|W|^{\frac{1}{p}}} \int_{0}^{|E|} \nu^{*}(t) \int_{0}^{|W|} \mu^{*}(s) \frac{1}{\max (s, t)^{\frac{1}{r}}} d s d t \\
& \lesssim \sup _{t>0} \frac{1}{t^{2+1 / p-1 / q}} \int_{0}^{t} \mu^{*}(s) d s \int_{0}^{t} \nu^{*}(s) d s \int_{0}^{t} \frac{1}{s^{\frac{1}{r}}} d s \lesssim \mathcal{G}
\end{aligned}
$$

for any sets $E$ and $W$ with positive measure, provided

$$
1<p, q, r<\infty, \quad \alpha+1 / r+1 / p>1, \quad \beta+1 / r+1 / q^{\prime}>1 .
$$


We note that the expression $\mathcal{G}$ depends only on $1 / p-1 / q$. Further, since conditions (4.4) contain strict inequalities, one can choose two pairs $\left(p_{0}, q_{0}\right)$ and $\left(p_{1}, q_{1}\right)$ such that $1<p_{0}<p<$ $p_{1}<\infty, 1<q_{0}<q<q_{1}<\infty$, and

$$
1 / p-1 / q=1 / p_{0}-1 / q_{0}=1 / p_{1}-1 / q_{1}, \quad \alpha+1 / r+1 / p_{i}>1, \quad \beta+1 / r+1 / q_{i}^{\prime}>1, \quad i=0,1 .
$$

Then, (4.2) holds for $\left(p_{0}, q_{0}\right)$ and $\left(p_{1}, q_{1}\right)$, and therefore,

$$
A: L^{p_{0}, 1} \rightarrow L^{q_{0}, \infty} \text { and } A: L^{p_{1}, 1} \rightarrow L^{q_{1}, \infty},
$$

where the norms are bounded by $\mathcal{G}\|K\|_{L^{r, \infty}}$ up to a constant. Then, by the interpolation theorem (see, e.g., [BS, Ch 5, §1]) we write

$$
A: L^{p(\theta), \tau_{1}} \rightarrow L^{q(\theta), \tau_{1}}
$$

and

$$
\|A\|_{L^{p(\theta), \tau_{1}} \rightarrow L^{q(\theta), \tau_{1}}} \leqslant C\left(\theta, p_{i}, q_{i}, r, \tau_{1}\right) \mathcal{G}\|K\|_{L^{r, \infty}}
$$

for

$$
1 / p(\theta)=(1-\theta) / p_{0}+\theta / p_{1}, \quad 1 / q(\theta)=(1-\theta) / q_{0}+\theta / q_{1}, \quad 0<\theta<1, \quad 0<\tau_{1} \leqslant \infty .
$$

For some $0<\theta<1$ we have $p(\theta)=p$. Since $1 / p_{0}-1 / q_{0}=1 / p_{1}-1 / q_{1}=1 / p(\theta)-1 / q(\theta)$, in this case $q(\theta)$ coincides with $q$.

Finally,

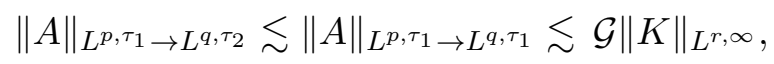

when $\tau_{1} \leqslant \tau_{2}$. Taking $p=\tau_{1}$ and $q=\tau_{2}$ implies (2.4).

Let us show necessity of (2.6) . Suppose (2.4) holds. For measurable sets $E$ and $W$ such that $|E|=|W|$, we put

$$
K(x):=|E-W|^{-\frac{1}{r}} \chi_{E-W}(x) .
$$

Then $\|K\|_{L^{r, \infty}\left(\mathbb{R}^{n}\right)}=1$. Using (4.3), we get

$$
\begin{aligned}
1 \gtrsim\|A\|_{L^{p}\left(\mathbb{R}^{n}\right) \rightarrow L^{q}\left(\mathbb{R}^{n}\right)} \gtrsim\|A\|_{L^{p, 1}\left(\mathbb{R}^{n}\right) \rightarrow L^{q, \infty}\left(\mathbb{R}^{n}\right)} & \gtrsim \frac{1}{|E|^{\frac{1}{q^{\prime}}}} \frac{1}{|W|^{\frac{1}{p}}}\left|\int_{E} \nu(y) \int_{W} K(y-x) \mu(x) d x d y\right| \\
& =\frac{1}{|E|^{1+\frac{1}{p}-\frac{1}{q}}} \frac{1}{|E-W|^{\frac{1}{r}}} \int_{E} \nu(y) \int_{W} \mu(x) d x d y .
\end{aligned}
$$

Taking supremum over all $E$ and $W$ completes the proof.

Proof of Corollary 1. First, we prove the sufficiency part. We take $\mu(x)=|x|^{-\alpha}$ and $\nu(x)=|x|^{-\beta}$ and note that (2.3) can be written as $\alpha / n+1 / r+1 / p>1$ and $\beta / n+1 / r+1 / q^{\prime}>1$. Further, $\mathcal{G}<\infty$ only if $\frac{1}{q}=\frac{1}{p}+\frac{1}{r}+\frac{\alpha+\beta}{n}-1$ and (2.7) follows.

Second, to prove necessity of condition (2.8) for a fixed $r \in(1, \infty)$, we consider $K(x)=|x|^{\gamma-n}$ such that $\frac{1}{r}=1-\frac{\gamma}{n}$. Therefore, $0<\gamma<n$ and $K \in L^{r, \infty}\left(\mathbb{R}^{n}\right)$. Then the boundedness of the fractional integral from $L_{\alpha}^{p}\left(\mathbb{R}^{n}\right)$ to $L_{-\beta}^{q}\left(\mathbb{R}^{n}\right)$ gives (see [Du, Th. 5.1]) that

$$
\frac{1}{q}=\frac{1}{p}+\frac{1}{r}+\frac{\alpha+\beta}{n}-1, \quad \alpha<n / p^{\prime}, \quad \beta<n / q .
$$

These conditions can be also verified using (2.6).

We have to show that $\alpha, \beta \geqslant 0$. Let an integer $N$ be sufficiently large. Putting $K(x):=$ $\chi_{B_{2}(\bar{N})}(x)$, we have $K \in L^{r, \infty}\left(\mathbb{R}^{n}\right), r>1$. If

$$
\|f * K\|_{L_{-\beta}^{q}\left(\mathbb{R}^{n}\right)} \lesssim\|f\|_{L_{\alpha}^{p}\left(\mathbb{R}^{n}\right)}\|K\|_{L^{r, \infty}\left(\mathbb{R}^{n}\right)}
$$


then the operator

$$
A_{K, \alpha, \beta} f(y)=\frac{1}{|y|^{\beta}} \int_{\mathbb{R}^{n}} K(x-y) f(x) \frac{d x}{|x|^{\alpha}}
$$

is bounded from $L^{p}\left(\mathbb{R}^{n}\right)$ to $L^{q}\left(\mathbb{R}^{n}\right)$. Taking into account (4.3),

$$
\begin{aligned}
1 \gtrsim\left\|A_{K, \alpha, \beta}\right\|_{L^{p}\left(\mathbb{R}^{n}\right) \rightarrow L^{q}\left(\mathbb{R}^{n}\right)} & \gtrsim\left\|A_{K, \alpha, \beta}\right\|_{L^{p, 1}\left(\mathbb{R}^{n}\right) \rightarrow L^{q, \infty}\left(\mathbb{R}^{n}\right)} \\
\asymp & \sup _{\substack{|E|>0\\
}} \frac{1}{|W|>\left.0\right|^{\frac{1}{q^{\prime}}}|W|^{\frac{1}{p}}}\left|\int_{E} \frac{1}{|y|^{\beta}} \int_{W} K(x-y) \frac{d x}{|x|^{\alpha}} d y\right| .
\end{aligned}
$$

Then, since $\beta<n / q<n$

$$
\begin{aligned}
& 1 \gtrsim \frac{1}{\left|B_{1}(\overline{0})\right|^{\frac{1}{q^{\prime}}}\left|B_{2}(\bar{N})\right|^{\frac{1}{p}}} \int_{B_{1}(\overline{0})} \frac{1}{|y|^{\beta}} \int_{B_{2}(\bar{N})} K(x-y) \frac{d x}{|x|^{\alpha}} d y \\
& \geqslant C_{p, q} \int_{B_{1}(\overline{0})}|y|^{-\beta} d y \int_{B_{1}(\bar{N})}|x|^{-\alpha} d x \gtrsim N^{-\alpha} .
\end{aligned}
$$

This gives $\alpha \geqslant 0$. Similarly, we get $\beta \geqslant 0$.

Finally, since

$$
L_{s}^{\theta}\left(\mathbb{R}^{n}\right) \subset L^{r, \infty}\left(\mathbb{R}^{n}\right) \quad \text { for } \quad \frac{1}{r}=\frac{s}{n}+\frac{1}{\theta}, \quad 0 \leqslant s<n / \theta^{\prime},
$$

(2.7) gives (1.2) provided (1.3), (1.4), and $\alpha, \beta, s \geqslant 0$.

\section{Boundedness of the weighted convolution operators in Lorentz spaces}

In this section we deal with Problem 2 and study the weighted convolution operator $A_{K}=$ $A_{K, \mu, \nu}$ given by (4.1). In particular, if $K(x)=|x|^{\gamma-n}$, then $A_{K}=A_{\gamma}$, where $A_{\gamma}$ is defined by (1.10). The next result gives sufficient conditions for the operator $A_{K}$ to be bounded from $L^{p, \tau_{1}}\left(\mathbb{R}^{n}\right)$ to $L^{q, \tau_{2}}\left(\mathbb{R}^{n}\right)$ in terms of $\mu, \nu$, and $K$. This implies sufficient conditions for the boundedness of the operator $A_{\gamma}$ given in Theorem 2 (A).

Theorem 3. Let $1<p, q<\infty, \quad 0<\tau_{1} \leqslant \tau_{2} \leqslant \infty$. Suppose either, (A) for any $\lambda \geqslant 1$,

$$
\mu^{*}(\lambda t) \lesssim \frac{\mu^{*}(t)}{\lambda^{\alpha}}, \quad \nu^{*}(\lambda t) \lesssim \frac{\nu^{*}(t)}{\lambda^{\beta}}, \quad K^{*}(\lambda t) \lesssim \frac{K^{*}(t)}{\lambda^{\rho}}, \quad t>0,
$$

where $\alpha \geqslant 0, \beta \geqslant 0, \rho \geqslant 0$, and

$$
\rho+\alpha+1 / p>1, \quad \rho+\beta+1 / q^{\prime}>1,
$$

or,

(B) for any $\lambda \geqslant 1$,

$$
\mu^{*}(t) \lesssim \mu^{*}(\lambda t) \lambda^{\bar{\alpha}}, \quad \nu^{*}(t) \lesssim \nu^{*}(\lambda t) \lambda^{\bar{\beta}}, \quad K^{*}(t) \lesssim K^{*}(\lambda t) \lambda^{\bar{\rho}}, \quad t>0,
$$

where $\bar{\alpha} \geqslant 0, \bar{\beta} \geqslant 0, \bar{\rho} \geqslant 0$, and

$$
\bar{\rho}+\bar{\alpha}+1 / p<1, \quad \bar{\rho}+\bar{\beta}+1 / q^{\prime}<1 .
$$


If

$$
\mathcal{D}:=\sup _{t>0} \frac{1}{t^{2+1 / p-1 / q}} \int_{0}^{t} \mu^{*}(s) d s \int_{0}^{t} \nu^{*}(s) d s \int_{0}^{t} K^{*}(s) d s<\infty,
$$

then the operator $A_{K}$ is bounded from $L^{p, \tau_{1}}\left(\mathbb{R}^{n}\right)$ to $L^{q, \tau_{2}}\left(\mathbb{R}^{n}\right)$ and

$$
\left\|A_{K}\right\|_{L^{p, \tau_{1}}\left(\mathbb{R}^{n}\right) \rightarrow L^{q, \tau_{2}}\left(\mathbb{R}^{n}\right)} \leqslant C \mathcal{D},
$$

where $C$ depends only on $p, q, \tau_{1}, \tau_{2}$ and the corresponding parameters from (5.1) or (5.2).

Proof. As it was shown in the proof of Theorem 1, it is sufficient to obtain the following estimate:

$$
\left\|A_{K}\right\|_{L^{p, 1}\left(\mathbb{R}^{n}\right) \rightarrow L^{q, \infty}\left(\mathbb{R}^{n}\right)} \lesssim \mathcal{D} .
$$

(A) Under conditions (5.1), following the proof of Theorem 1, we have

$$
\left\|A_{K}\right\|_{L^{p, 1}\left(\mathbb{R}^{n}\right) \rightarrow L^{q, \infty}\left(\mathbb{R}^{n}\right)} \sup _{|E|>0,|W|>0} \frac{1}{|E|^{\frac{1}{q^{\prime}}}} \frac{1}{|W|^{\frac{1}{p}}} \int_{0}^{|E|} \nu^{*}(t) \int_{0}^{|W|} \mu^{*}(s) K^{* *}(\max (s, t)) d s d t \lesssim \mathcal{D},
$$

where the last estimate directly follows from Lemma 3.

(B) Suppose conditions (5.2) hold. Let us verify that

$$
\sup _{|E|>0,|W|>0} \frac{1}{|E|^{\frac{1}{q^{\prime}}}} \frac{1}{|W|^{\frac{1}{p}}} \int_{0}^{|E|} \nu^{*}(t) \int_{0}^{|W|} \mu^{*}(s) K^{* *}(\max (s, t)) d s d t \lesssim \mathcal{D} .
$$

Let $0<|W|<|E|$ and $|E| /|W|=: \lambda>1$. Note that

$$
K^{* *}\left(\max \left(\frac{s}{\lambda}, t\right)\right) \lesssim \lambda^{\bar{\rho}} K^{* *}(\max (s, t)) \quad \text { for any } \quad s, t>0 .
$$

Indeed, using monotonicity of $K^{* *}$ and condition (5.2), we get

$$
\begin{aligned}
K^{* *}(\max (s / \lambda, t)) & =\frac{1}{\max (s / \lambda, t)} \int_{0}^{\max (s / \lambda, t)} K^{*}(u) d u \\
& \leqslant \frac{\lambda}{\max (s, t)} \int_{0}^{\max (s, t) / \lambda} K^{*}(u) d u \\
& =\frac{1}{\max (s, t)} \int_{0}^{\max (s, t)} K^{*}\left(\frac{u}{\lambda}\right) d u \leqslant C \lambda^{\bar{\rho}} K^{* *}(\max (s, t)) .
\end{aligned}
$$

In view of $\bar{\alpha}+\bar{\rho}<\frac{1}{p^{\prime}}$, we have

$$
\begin{aligned}
& \frac{1}{|E|^{\frac{1}{q^{\prime}}}} \frac{1}{|W|^{\frac{1}{p}}} \int_{0}^{|E|} \nu^{*}(t) \int_{0}^{|W|} \mu^{*}(s) K^{* *}(\max (s, t)) d s d t \\
& =\frac{\lambda^{\frac{1}{p}-1}}{|E|^{\frac{1}{q^{\prime}}+\frac{1}{p}}} \int_{0}^{|E|} \nu^{*}(t) \int_{0}^{|E|} \mu^{*}\left(\frac{s}{\lambda}\right) K^{* *}\left(\max \left(\frac{s}{\lambda}, t\right)\right) d s d t \\
& \lesssim \frac{\lambda^{\bar{\alpha}+\bar{\rho}+\frac{1}{p}-1}}{|E|^{\frac{1}{q^{\prime}}+\frac{1}{p}}} \int_{0}^{|E|} \nu^{*}(t) \int_{0}^{|E|} \mu^{*}(s) K^{* *}(\max (s, t)) d s d t \\
& \leqslant \frac{1}{|E|^{\frac{1}{q^{\prime}}+\frac{1}{p}}} \int_{0}^{|E|} \nu^{*}(t) \int_{0}^{|E|} \mu^{*}(s) K^{* *}(\max (s, t)) d s d t .
\end{aligned}
$$


Further, Lemma 2 implies

$$
\frac{1}{|E|^{\frac{1}{q^{\prime}}}} \frac{1}{|W|^{\frac{1}{p}}} \int_{0}^{|E|} \nu^{*}(t) \int_{0}^{|W|} \mu^{*}(s) K^{* *}(\max (s, t)) d s d t \lesssim \mathcal{D} .
$$

The case $|W|>|E|$ is similar. Thus, we have shown (5.3), which concludes the proof.

\section{Proof of Theorem 2 and its corollaries}

Proof of Theorem 2. We start with (B). If $A_{\gamma}$ is bounded from $L^{p, \tau_{1}}\left(\mathbb{R}^{n}\right)$ to $L^{q, \tau_{2}}\left(\mathbb{R}^{n}\right)$, then it is $(p, q)$ weak-type operator, i.e., $A_{\gamma}: L^{p, 1}\left(\mathbb{R}^{n}\right) \rightarrow L^{q, \infty}\left(\mathbb{R}^{n}\right)$, and therefore, by (4.3), we have

$$
\begin{aligned}
& \left\|A_{\gamma}\right\|_{L^{p, \tau_{1}}\left(\mathbb{R}^{n}\right) \rightarrow L^{q, \tau_{2}\left(\mathbb{R}^{n}\right)}} \gtrsim\left\|A_{\gamma}\right\|_{L^{p, \min \left(1, \tau_{1}\right)}\left(\mathbb{R}^{n}\right) \rightarrow L^{q, \infty}\left(\mathbb{R}^{n}\right)} \asymp\left\|A_{\gamma}\right\|_{L^{p, 1}\left(\mathbb{R}^{n}\right) \rightarrow L^{q, \infty}\left(\mathbb{R}^{n}\right)} \\
& \asymp \sup _{|E|>0,|W|>0} \frac{1}{|E|^{1 / q^{\prime}}} \frac{1}{|W|^{1 / p}} \int_{E} \nu(y) \int_{W} \mu(x)|x-y|^{\gamma-n} d x d y \\
& \geqslant \sup _{B \in M} \frac{1}{|B|^{\frac{1}{p}+\frac{1}{q^{\gamma}}}} \int_{B} \nu(y) \int_{B} \mu(x)|x-y|^{\gamma-n} d x d y \\
& \gtrsim \sup _{B \in M} \frac{1}{|B|^{2+\frac{1}{p}-\frac{1}{q}-\frac{\gamma}{n}}} \int_{B} \nu(x) d x \int_{B} \mu(x) d x=\mathcal{F},
\end{aligned}
$$

that is, (2.11) is true.

The part (A) follows from Theorem 3 (A) for $K(x)=|x|^{\gamma-n}$. Taking $\rho=1-\gamma / n$ implies

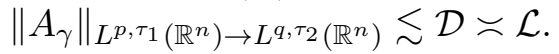

Proof of Corollary 2, To show that $\mathcal{L} \lesssim \mathcal{F}$ it is enough to verify that, for a fixed positive $a$,

$$
\sup _{|E|=a^{n}} \int_{E} \nu_{0}(|x|) d x \lesssim \int_{B_{a_{1}}(\overline{0})} \nu_{0}(|x|) d x
$$

and

$$
\sup _{|W|=a^{n}} \int_{W} \mu_{0}(|x|) d x \lesssim \int_{B_{a_{1}}(\overline{0})} \mu_{0}(|x|) d x
$$

where $a_{1} / a=C(n)$.

Let us show the first inequality. We have

$$
\sup _{|E|=a^{n}} \int_{E} \nu_{0}(|x|) d x=\int_{0}^{a^{n}}\left(\nu_{0}(|\cdot|)\right)^{*}(t) d t \lesssim \int_{0}^{a_{1}^{n}} \nu_{0}^{*}\left(t^{1 / n}\right) d t \lesssim \int_{0}^{a_{1}} \nu_{0}^{*}(t) t^{n-1} d t .
$$

Using condition (2.12), we get

$$
\begin{aligned}
\sup _{|E|=a^{n}} \int_{E} \nu_{0}(|x|) d x & \lesssim \int_{0}^{a_{1}}\left(\frac{1}{t} \int_{t / 2}^{t} \nu_{0}(s) d s\right) t^{n-1} d t \\
& \lesssim \int_{0}^{a_{1}} \nu_{0}(t) t^{n-1} d t \lesssim \int_{B_{a_{1}}(\overline{0})} \nu_{0}(|x|) d x
\end{aligned}
$$


i.e., (6.1) follows. Using (6.1) and (6.2) we get

$$
\begin{aligned}
\mathcal{L} & =\sup _{a>0} \sup _{|E|=|W|=a} \frac{1}{a^{2+\frac{1}{p}-\frac{1}{q}-\frac{\gamma}{n}}} \int_{W} \mu_{0}(|x|) d x \int_{E} \nu_{0}(|x|) d x \\
& \lesssim \sup _{r>0} \frac{1}{\left|B_{r}(\overline{0})\right|^{2+\frac{1}{p}-\frac{1}{q}-\frac{\gamma}{n}}} \int_{B_{r}(\overline{0})} \mu_{0}(|x|) d x \int_{B_{r}(\overline{0})} \nu_{0}(|x|) d x \leqslant \mathcal{F} .
\end{aligned}
$$

Finally, in view of $\mathcal{F} \leqslant \mathcal{L} \lesssim \mathcal{F}$, the result follows from Theorem 2 ,

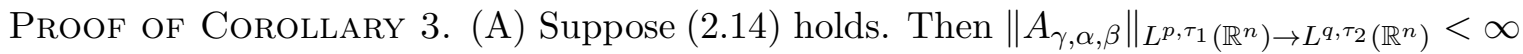
follows from Corollary 2, Indeed, for $\mu(x)=|x|^{-\alpha}$ and $\nu(x)=|x|^{-\beta}$, we have $A_{\gamma, \alpha, \beta}=A_{\gamma, \mu(\cdot), \nu(\cdot)}$. Since conditions (2.14) are equivalent to

$$
\sup _{a>0} \frac{1}{a^{n\left(2+\frac{1}{p}-\frac{1}{q}-\frac{\gamma}{n}\right)}} \int_{0}^{a} \nu_{0}(r) r^{n-1} d r \int_{0}^{a} \mu_{0}(r) r^{n-1} d r<\infty
$$

and

$$
\left.\frac{\alpha}{n}+\frac{1}{p}>\frac{\gamma}{n}, \quad \frac{\beta}{n}+\frac{1}{q^{\prime}}>\frac{\gamma}{n}, \quad \text { (cf. (2.9) }\right),
$$

(2.13) implies that $A_{\gamma, \alpha, \beta}: L^{p, \tau_{1}}\left(\mathbb{R}^{n}\right) \rightarrow L^{q, \tau_{2}}\left(\mathbb{R}^{n}\right)$.

Let now the operator $A_{\gamma, \alpha, \beta}$ be bounded from $L^{p, \tau_{1}}\left(\mathbb{R}^{n}\right)$ to $L^{q, \tau_{2}}\left(\mathbb{R}^{n}\right)$. Then (2.13) gives that $\gamma=\alpha+\beta+n\left(\frac{1}{p}-\frac{1}{q}\right)$.

First, we assume that $1<\tau_{1} \leqslant \tau_{2} \leqslant \infty$ and $\tau_{1} \neq \infty$. Let us show that $\alpha<n / p^{\prime}$ and $\beta<n / q$. Using [NT2, Th. 4.1] and taking into account that $\left(L^{p, \tau_{1}}\left(\mathbb{R}^{n}\right)\right)^{\prime}=L^{p^{\prime}, \tau_{1}^{\prime}}\left(\mathbb{R}^{n}\right)$, we get

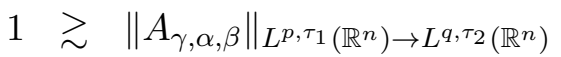

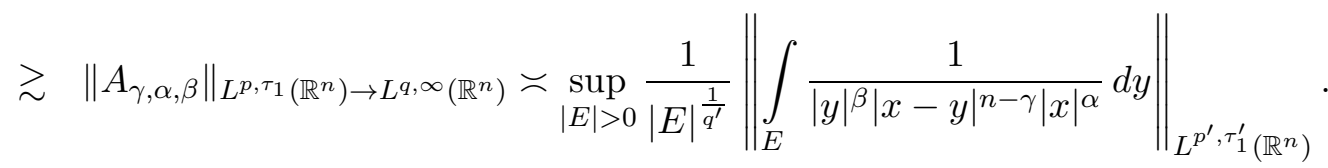

We further choose $E$ to be $B_{1}(\overline{0})$ or $B_{1}(\overline{3})$ and we get

$$
\begin{aligned}
& 1 \gtrsim \max \left\{\frac{1}{\left|B_{1}(\overline{0})\right|^{\frac{1}{q^{\prime}}}}\left\|\chi_{\{x:|x|>2\}}(x) \int_{B_{1}(\overline{0})} \frac{1}{|y|^{\beta}|x-y|^{n-\gamma}|x|^{\alpha}} d y\right\|_{L^{p^{\prime}, \tau_{1}^{\prime}\left(\mathbb{R}^{n}\right)}},\right. \\
& \left.\frac{1}{\left|B_{1}(3)\right|^{\frac{1}{q^{\prime}}}}\left\|\chi_{\{x:|x|<1\}}(x) \int_{B_{1}(\overline{3})} \frac{1}{|y|^{\beta}|x-y|^{n-\gamma}|x|^{\alpha}} d y\right\|_{L^{p^{\prime}, \tau_{1}^{\prime}\left(\mathbb{R}^{n}\right)}}\right\} \\
& \gtrsim \max \left\{\left\|\chi_{\{x:|x|>2\}}(x)|x|^{\gamma-n-\alpha}\right\|_{L^{p^{\prime}, \tau_{1}^{\prime}\left(\mathbb{R}^{n}\right)}},\left\|\chi_{\{x:|x|<1\}}(x)|x|^{-\alpha}\right\|_{L^{p^{\prime}, \tau_{1}^{\prime}\left(\mathbb{R}^{n}\right)}}\right\} \\
& \gtrsim \max \left\{\left(\int_{1}^{\infty} t^{\left(1 / p^{\prime}-1+\gamma / n-\alpha / n\right) \tau_{1}^{\prime}} \frac{d t}{t}\right)^{\frac{1}{\tau_{1}^{\prime}}},\left(\int_{0}^{1} t^{\left(1 / p^{\prime}-\alpha / n\right) \tau_{1}^{\prime}} \frac{d t}{t}\right)^{\frac{1}{\tau_{1}^{\prime}}}\right\} .
\end{aligned}
$$

Taking into account that $\tau_{1}^{\prime}<\infty$ and $\gamma=\alpha+\beta+n\left(\frac{1}{p}-\frac{1}{q}\right)$, we have that convergence of integrals implies $\beta<n / q$ and $\alpha<n / p^{\prime}$. 
The case $\tau_{1}=\tau_{2}=\infty$ follows from the proof above and

$$
\left\|A_{\gamma, \alpha, \beta}\right\|_{L^{p, \infty}\left(\mathbb{R}^{n}\right) \rightarrow L^{q, \infty}\left(\mathbb{R}^{n}\right)} \geqslant\left\|A_{\gamma, \alpha, \beta}\right\|_{L^{p, 2}\left(\mathbb{R}^{n}\right) \rightarrow L^{q, \infty}\left(\mathbb{R}^{n}\right)} .
$$

Let now $0<\tau_{1} \leqslant \tau_{2}<\infty$. Since the function $f(x)=\left(\tau_{1} / p\right)^{1 / \tau_{1}}|W|^{-1 / p} \chi_{W}(x)$ satisfies $\|f\|_{L^{p, \tau_{1}\left(\mathbb{R}^{n}\right)}}=1$, we get

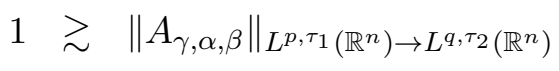

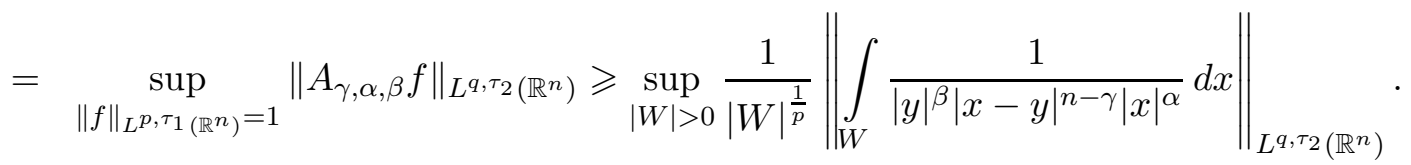

Repeating the arguments gives $\beta<n / q$ and $\alpha<n / p^{\prime}$.

(B). Let $0<\tau \leqslant 1$ and (2.15) be true. Since

$$
\begin{aligned}
\left\|A_{\gamma, \alpha, \beta}\right\|_{L^{p, \tau}\left(\mathbb{R}^{n}\right) \rightarrow L^{q, \infty}\left(\mathbb{R}^{n}\right)} & \asymp\left\|A_{\gamma, \alpha, \beta}\right\|_{L^{p, 1}\left(\mathbb{R}^{n}\right) \rightarrow L^{q, \infty}\left(\mathbb{R}^{n}\right)} \\
& \asymp \sup _{|E|>0,|W|>0} \frac{1}{|E|^{1 / q^{\prime}}} \frac{1}{|W|^{1 / p}} \int_{E} \int_{W} \frac{1}{|y|^{\beta}|x-y|^{n-\gamma}|x|^{\alpha}} d x d y,
\end{aligned}
$$

using Lemmas 1 and 3 , we get $\left\|A_{\gamma, \alpha, \beta}\right\|_{L^{p, 1}\left(\mathbb{R}^{n}\right) \rightarrow L^{q, \infty}\left(\mathbb{R}^{n}\right)} \lesssim \mathcal{D}$, where $\mathcal{D}$ is defined in Lemma 3 and $\mu(x)=|x|^{-\alpha}, \nu(x)=|x|^{-\beta}$, and $K(x)=|x|^{\gamma-n}$. Note that conditions (3.4)-(3.5) hold since $\alpha / n \leqslant 1 / p^{\prime}$ and $\beta / n \leqslant 1 / q$. Finally, $\left\|A_{\gamma, \alpha, \beta}\right\|_{L^{p, 1}\left(\mathbb{R}^{n}\right) \rightarrow L^{q, \infty}\left(\mathbb{R}^{n}\right)} \lesssim \mathcal{D}<\infty$ because of $\gamma=\alpha+\beta+n\left(\frac{1}{p}-\frac{1}{q}\right)$

To prove the inverse part, we have

$$
\begin{aligned}
& 1 \gtrsim\left\|A_{\gamma, \alpha, \beta}\right\|_{L^{p, 1}\left(\mathbb{R}^{n}\right) \rightarrow L^{q, \infty}\left(\mathbb{R}^{n}\right)} \\
& \gtrsim \max \left\{\sup _{0<\delta \leqslant 1} \frac{1}{\left|B_{\delta}(\overline{0})\right|^{\frac{1}{q^{\prime}}}\left|B_{1}(\overline{3})\right|^{\frac{1}{p}}} \int_{B_{1}(\overline{3})} \int_{B_{\delta}(\overline{0})} \frac{d x d y}{|y|^{\beta}|x-y|^{n-\gamma}|x|^{\alpha}},\right. \\
& \left.\sup _{0<\delta \leqslant 1} \frac{1}{\left|B_{1}(\overline{3})\right|^{\frac{1}{q^{\prime}}}\left|B_{\delta}(\overline{0})\right|^{\frac{1}{p}}} \int_{B_{\delta}(\overline{0})} \int_{B_{1}(\overline{3})} \frac{d x d y}{|y|^{\beta}|x-y|^{n-\gamma}|x|^{\alpha}}\right\} \\
& \gtrsim \max \left\{\sup _{0<\delta \leqslant 1} \frac{1}{\left|B_{\delta}(\overline{0})\right|^{\frac{1}{q^{\prime}}}} \int_{B_{\delta}(\overline{0})} \frac{1}{|y|^{\beta}} d y, \sup _{0<\delta \leqslant 1} \frac{1}{\left|B_{\delta}(\overline{0})\right|^{\frac{1}{p}}} \int_{B_{\delta}(\overline{0})} \frac{1}{|x|^{\alpha}} d x\right\} .
\end{aligned}
$$

This gives $\alpha / n \leqslant 1 / p^{\prime}$ and $\beta / n \leqslant 1 / q$. Necessity of the condition $\gamma=\alpha+\beta+n\left(\frac{1}{p}-\frac{1}{q}\right)$ follows from

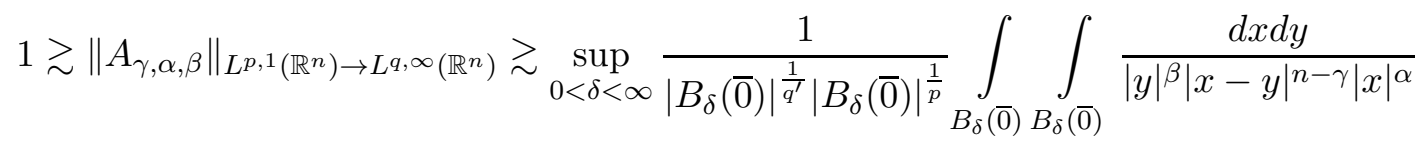

and the homogeneity argument.

\section{Final remarks}

1. Theorem A provides sufficient conditions for weighted Young's inequality $L_{\alpha}^{p}\left(\mathbb{R}^{n}\right) *$ $L_{s}^{\theta}\left(\mathbb{R}^{n}\right) \subset L_{-\beta}^{q}\left(\mathbb{R}^{n}\right)$ to hold. Certain necessary conditions, which differ from sufficient ones, 
were obtained by Bui Bu1, Th. 2.1]. He posed the problem ([Bu1, p.32]) of finding necessary and sufficient conditions for weighted Young's inequality. We give a solution of this problem.

Theorem A'. Suppose $1<p, q<\infty$ and $1<\theta \leqslant \infty$. Then

$$
L_{\alpha}^{p}\left(\mathbb{R}^{n}\right) * L_{s}^{\theta}\left(\mathbb{R}^{n}\right) \subset L_{-\beta}^{q}\left(\mathbb{R}^{n}\right), \quad \frac{1}{q} \leqslant \frac{1}{p}+\frac{1}{\theta},
$$

if and only if

$(7.3)^{\prime}$

$$
\begin{gathered}
\frac{1}{q}=\frac{1}{p}+\frac{1}{\theta}+\frac{\alpha+\beta+s}{n}-1, \\
\alpha<n / p^{\prime}, \quad \beta<n / q, \quad s<n / \theta^{\prime} \quad \text { if } \theta<\infty \\
\alpha<n / p^{\prime}, \quad \beta<n / q, \quad 0<s<n \quad \text { if } \theta=\infty
\end{gathered}
$$

and

$$
\alpha+\beta \geqslant 0, \quad \alpha+s \geqslant 0, \quad \beta+s \geqslant 0 .
$$

REmark. Necessity of condition (7.4) was recently proved in [MV, Rem. 4.4] and [DD, Rem. 2.1]. Our proof uses different argument. Note also that in case of $\theta=\infty$ condition (7.4) is equivalent to the following condition:

$$
\alpha+\beta \geqslant 0 .
$$

This follows from (7.2) and (7.3) $)^{\prime}$.

Proof. Sufficiency for the case $\theta<\infty$ was proved by Kerman (Theorem A). For the case $\theta=\infty$ see [Ke, Th. 3.4], [Bu1, Th. 2.2 (ii)], and [Be, Sect. 3].

Let us prove the necessity part. First, by scaling argument, we have necessity of condition (7.2) (see [Bu1, Th. 2.1]).

Second, let $1<\theta \leqslant \infty$ and (17.1) hold true. Take $\widetilde{K}(x)=K(x)|x|^{s} \in L^{\theta}\left(\mathbb{R}^{n}\right)$, i.e., $K \in$ $L_{s}^{\theta}\left(\mathbb{R}^{n}\right)$. Then the operator

$$
A_{\tilde{K}, \alpha, \beta, s} f(y)=\frac{1}{|y|^{\beta}} \int_{\mathbb{R}^{n}} \frac{\widetilde{K}(x-y) f(x)}{|x-y|^{s}|x|^{\alpha}} d x
$$

is bounded from $L^{p}\left(\mathbb{R}^{n}\right)$ to $L^{q}\left(\mathbb{R}^{n}\right)$. Using [NT2, Th. 4.1], we get

$$
\begin{aligned}
\left\|A_{\tilde{K}, \alpha, \beta, s}\right\|_{L^{p}\left(\mathbb{R}^{n}\right) \rightarrow L^{q}\left(\mathbb{R}^{n}\right)} & \geqslant\left\|A_{\tilde{K}, \alpha, \beta, s}\right\|_{L^{p}\left(\mathbb{R}^{n}\right) \rightarrow L^{q, \infty}\left(\mathbb{R}^{n}\right)} \\
& \asymp \sup _{|E|>0} \frac{1}{|E|^{\frac{1}{q^{\prime}}}}\left\|\int_{E} \frac{\widetilde{K}(x-y)}{|y|^{\beta}|x-y|^{s}|x|^{\alpha}} d y\right\|_{\left(L^{p}\left(\mathbb{R}^{n}\right)\right)^{\prime}} \\
& \gtrsim \sup _{|E|>0} \frac{1}{|E|^{\frac{1}{q^{\prime}}}}\left(\int_{\mathbb{R}^{n}}\left|\int \frac{\widetilde{K}(x-y)}{|y|^{\beta}|x-y|^{s}|x|^{\alpha}} d y\right|^{p^{\prime}} d x\right)^{1 / p^{\prime}} .
\end{aligned}
$$

Let an integer $N$ be sufficiently large. Put

$$
\widetilde{K}(x):=\chi_{B_{2}(\bar{N})}(x) \in L^{\theta}\left(\mathbb{R}^{n}\right) .
$$


Then

$$
\begin{aligned}
1 & \gtrsim\left\|A_{\tilde{K}, \alpha, \beta, s}\right\|_{L^{p}\left(\mathbb{R}^{n}\right) \rightarrow L^{q}\left(\mathbb{R}^{n}\right)} \gtrsim \frac{1}{\left|B_{1}(-\bar{N})\right|^{\frac{1}{q^{\prime}}}}\left(\int_{B_{1}(\overline{0})}\left|\int_{B_{1}(-\bar{N})} \frac{\tilde{K}(x-y)}{|y|^{\beta}|x-y|^{s}|x|^{\alpha}} d y\right|^{p^{\prime}} d x\right)^{1 / p^{\prime}} \\
& \gtrsim\left(\int_{B_{1}(\overline{0})}|x|^{-\alpha p^{\prime}} d x\right)^{1 / p^{\prime}} \int_{B_{1}(\bar{N})} \frac{d y}{|y|^{s+\beta}} .
\end{aligned}
$$

In the last expression, the first integral converges only if $\alpha<n / p^{\prime}$ while the second integral is equivalent to $N^{-(\beta+s)}$ which is bounded by a constant only if $\beta+s \geqslant 0$.

Taking into account that

$$
\left\|A_{\tilde{K}, \alpha, \beta, s}\right\|_{L^{p}\left(\mathbb{R}^{n}\right) \rightarrow L^{q}\left(\mathbb{R}^{n}\right)}=\left\|A_{\tilde{K}, \beta, \alpha, s}\right\|_{L^{q^{\prime}}\left(\mathbb{R}^{n}\right) \rightarrow L^{p^{\prime}}\left(\mathbb{R}^{n}\right)},
$$

we also get that $\alpha+s \geqslant 0$ and $\beta<n / q$.

To prove necessity of conditions $\alpha+\beta \geqslant 0$ and $s<n / \theta^{\prime}$, we consider the operator

$$
\left(A_{\tilde{f}, \alpha, \beta, s} K\right)(y)=\frac{1}{|y|^{\beta}} \int_{\mathbb{R}^{n}} \frac{\tilde{f}(x-y) K(x)}{|x-y|^{\alpha}|x|^{s}} d x
$$

from $L^{\theta}\left(\mathbb{R}^{n}\right)$ to $L^{q}\left(\mathbb{R}^{n}\right)$ with the kernel

$$
\frac{\tilde{f}(x-y)}{|y|^{\beta}|x-y|^{\alpha}|x|^{s}}
$$

where $\tilde{f}(x)=f(x)|x|^{\alpha} \in L^{p}\left(\mathbb{R}^{n}\right)$, and proceed similarly.

Finally, we have to show that if $\theta=\infty$, then $s>0$. Let $L_{\alpha}^{p}\left(\mathbb{R}^{n}\right) * L_{s}^{\infty}\left(\mathbb{R}^{n}\right) \subset L_{-\beta}^{q}\left(\mathbb{R}^{n}\right)$, $1<p \leqslant q<\infty$. We define

$$
K(x):=|x|^{-s} \in L_{s}^{\infty}\left(\mathbb{R}^{n}\right), \quad f(x):=\chi_{\mathbb{R}^{n} \backslash B_{2}(\overline{0})}(x)|x|^{-\alpha-n / p}(\ln |x|)^{-2 / p} \in L_{\alpha}^{p}\left(\mathbb{R}^{n}\right) .
$$

Taking into account that $\alpha<n / p^{\prime}$, we notice that $s$ has to be positive in order that the integral

$$
(K * f)(y)=\int_{\mathbb{R}^{n} \backslash B_{2}(\overline{0})} \frac{1}{|y-x|^{s}|x|^{\alpha+n / p}(\ln |x|)^{2 / p}} d x
$$

converges.

2. The Plancherel-Polya-Nikol'skii inequality plays an important role in the theory of function spaces (see, e.g., $\mathbf{M V}, \mathbf{T r}$ ) and approximation theory (see, e.g., $\mathbf{D L}, \mathbf{D T}]$ ). The following result provides sufficient conditions for a two-weight version of this inequality, partially answering the question posed in $\mathbf{M V}$, Rem. 4.2]. Let $\mathcal{F}$ and $\mathcal{F}^{-1}$ denote the Fourier transform and its inverse.

COROLlary 4. Let $p, q, r, \mu$, and $\nu$ satisfy conditions of Theorem 1. Let $Q_{d}$ is a rectangular parallelepiped in $\mathbb{R}^{n}$ with edges of lengths $d=\left(d_{1}, \ldots, d_{n}\right)$. If $\operatorname{supp}(\mathcal{F} f) \subseteq Q_{d}$, then

$$
\|f\|_{L^{q}\left(\nu ; \mathbb{R}^{n}\right)} \lesssim \mathcal{G}\left(\prod_{j=1}^{n} d_{j}\right)^{1 / r^{\prime}}\|f\|_{L^{p}\left(\mu^{-1} ; \mathbb{R}^{n}\right)}
$$


where $\mathcal{G}$ is given by (2.5).

REMARK. For the power weights necessary and sufficient conditions for the Plancherel-PolyaNikol'skii inequality were proved in [MV], see Proposition 4.1 and Remark 4.2. PlancherelPolya-Nikol'skii's inequality with $A_{\infty}$ weights were studies in $[\mathbf{B u 2}, \mathbf{M T}$. The weighted version of Nikol'skii's inequality for trigonometric polynomials was also studied in [NT2, Cor. 5.2].

The proof of Corollary 4 immediately follows from the following result.

COROLlary 5. Let $Q_{d}$ be a rectangular parallelepiped in $\mathbb{R}^{n}$ with edges of lengths $d=$ $\left(d_{1}, \ldots, d_{n}\right)$ and

$$
S_{Q_{d}}(f):=\mathcal{F}^{-1} \chi_{Q_{d}} \mathcal{F} f
$$

(A). Let $p, q, r$ and $\mu, \nu$ satisfy conditions of Theorem 1. If $\mathcal{G}<\infty$, where $\mathcal{G}$ is defined by (2.5), then

$$
\left\|S_{Q_{d}}\right\|_{L^{p}\left(\mu^{-1} ; \mathbb{R}^{n}\right) \rightarrow L^{q}\left(\nu ; \mathbb{R}^{n}\right)} \leqslant C \mathcal{G}\left(\prod_{j=1}^{n} d_{j}\right)^{1 / r^{\prime}}
$$

where $C$ is independent of $d$.

(B). Let $1<p, q, r<\infty$. If

$$
\left\|S_{Q_{d}}\right\|_{L^{p}\left(\mu^{-1} ; \mathbb{R}^{n}\right) \rightarrow L^{q}\left(\nu ; \mathbb{R}^{n}\right)}<\infty
$$

then

$$
\mathcal{K}:=\sup _{\frac{\pi}{4 d} \leqslant t \leqslant \frac{\pi}{2 d}} \sup _{Q_{t}} \frac{\nu\left(Q_{t}\right) \mu\left(Q_{t}\right)}{\left|Q_{t}\right|^{1+\frac{1}{r}+\frac{1}{p}-\frac{1}{q}}}<\infty
$$

and

$$
\left\|S_{Q_{d}}\right\|_{L^{p}\left(\mu^{-1} ; \mathbb{R}^{n}\right) \rightarrow L^{q}\left(\nu ; \mathbb{R}^{n}\right)} \geqslant C \mathcal{K}\left(\prod_{j=1}^{n} d_{j}\right)^{1 / r^{\prime}}
$$

where $C$ is independent of $d$.

Proof. Taking into account that $S_{Q_{d}}(f)=\left(\mathcal{F}^{-1} \chi_{Q_{d}}\right) * f$, and

$$
\mathcal{F}^{-1} \chi_{Q_{d}}(x)=\prod_{j=1}^{n} \frac{\sin \left(d_{j} x_{j} / 2\right)}{x_{j} / 2}
$$

we get from Theorem 1 that

$$
\begin{aligned}
\left\|S_{Q_{d}}(f)\right\|_{L^{q}\left(\nu ; \mathbb{R}^{n}\right)} & \lesssim \mathcal{G}\left\|\prod_{j=1}^{n} \frac{\sin \left(d_{j} x_{j} / 2\right)}{x_{j} / 2}\right\|_{L^{r, \infty}\left(\mathbb{R}^{n}\right)}\|f\|_{L^{p}\left(\mu^{-1} ; \mathbb{R}^{n}\right)} \\
& \lesssim \mathcal{G}\left(\prod_{j=1}^{n} d_{j}\right)^{\frac{1}{r^{\prime}}}\|f\|_{L^{p}\left(\mu^{-1} ; \mathbb{R}^{n}\right)},
\end{aligned}
$$

i.e., (7.5) follows.

Let now $\left\|S_{Q_{d}}\right\|_{L^{p}\left(\mu^{-1} ; \mathbb{R}^{n}\right) \rightarrow L^{q}\left(\nu ; \mathbb{R}^{n}\right)}<\infty$. Then, defining the operator

$$
S_{Q_{d}, \mu, \nu}(f, y)=\nu(y) \int_{\mathbb{R}^{n}} \prod_{j=1}^{n} \frac{\sin \left(d_{j}\left(y_{j}-x_{j}\right) / 2\right)}{\left(y_{j}-x_{j}\right) / 2} \mu(x) f(x) d x,
$$


we get

$$
\begin{aligned}
& \left\|S_{Q_{d}}\right\|_{L^{p}\left(\mu^{-1} ; \mathbb{R}^{n}\right) \rightarrow L^{q}\left(\nu ; \mathbb{R}^{n}\right)}=\left\|S_{Q_{d}, \mu, \nu}\right\|_{L^{p}\left(\mathbb{R}^{n}\right) \rightarrow L^{q}\left(\mathbb{R}^{n}\right)} \gtrsim\left\|S_{Q_{d}, \mu, \nu}\right\|_{L^{p, 1}\left(\mathbb{R}^{n}\right) \rightarrow L^{q, \infty}\left(\mathbb{R}^{n}\right)} \\
& \asymp \sup _{|E|>0,|W|>0} \frac{1}{|E|^{1 / q^{\prime}}} \frac{1}{|W|^{1 / p}}\left|\int_{E} \nu(y) \int_{W} \mu(x) \prod_{j=1}^{n} \frac{\sin \left(d_{j}\left(y_{j}-x_{j}\right) / 2\right)}{\left(y_{j}-x_{j}\right) / 2} d x d y\right| .
\end{aligned}
$$

Since for $x, y \in Q_{t}$ we have that $y-x \in Q_{2 t}(\overline{0})$, where $Q_{2 t}(\overline{0})$ is the rectangular parallelepiped with edges of lengths $2 t=\left(2 t_{1}, \ldots, 2 t_{n}\right)$ centered at $\overline{0}=(0, \ldots, 0)$, then

$$
\begin{aligned}
\left\|S_{Q_{d}}\right\|_{L^{p}\left(\mu^{-1} ; \mathbb{R}^{n}\right) \rightarrow L^{q}\left(\nu ; \mathbb{R}^{n}\right)} & \gtrsim \sup _{\frac{\pi}{4 d} \leqslant t \leqslant \frac{\pi}{2 d}} \sup _{Q_{t}} \frac{1}{\left|Q_{t}\right|^{\frac{1}{p}+\frac{1}{q^{\prime}}}} \int_{Q_{t}} \nu(y) \int_{Q_{t}} \mu(x) \prod_{j=1}^{n} \frac{\sin \left(d_{j}\left(y_{j}-x_{j}\right) / 2\right)}{\left(y_{j}-x_{j}\right) / 2} d x d y \\
& \gtrsim \prod_{j=1}^{n} d_{j} \sup _{\frac{\pi}{4 d} \leqslant t \leqslant \frac{\pi}{2 d}} \sup _{Q_{t}} \frac{1}{\left|Q_{t}\right|^{\frac{1}{p}+\frac{1}{q^{\prime}}}} \int_{Q_{t}} \nu(y) \int_{Q_{t}} \mu(x) d x d y \\
& \gtrsim\left(\prod_{j=1}^{n} d_{j}\right)^{1 / r^{\prime}} \sup _{\frac{\pi}{4 d} \leqslant t \leqslant \frac{\pi}{2 d}} \sup _{Q_{t}} \frac{\nu\left(Q_{t}\right) \mu\left(Q_{t}\right)}{\left|Q_{t}\right|^{1+\frac{1}{r}+\frac{1}{p}-\frac{1}{q}}} .
\end{aligned}
$$

For power weights $\mu(x)=|x|^{-\alpha}$ and $\nu(x)=|x|^{-\beta}$, we obtain necessary and sufficient conditions for boundedness of the operator $S_{Q_{d}}$.

Corollary 6. Let $1<p, q, s<\infty$ and $\alpha \geqslant 0, \beta \geqslant 0$. Then

$$
\left\|S_{Q_{d}}(f)\right\|_{L_{-\beta}^{q}\left(\mathbb{R}^{n}\right)} \leqslant C\left(\prod_{j=1}^{n} d_{j}\right)^{1 / s}\|f\|_{L_{\alpha}^{p}\left(\mathbb{R}^{n}\right)} \quad \forall \quad d_{1}, \ldots, d_{n}>0,
$$

where $Q_{d}$ is a rectangular parallelepiped in $\mathbb{R}^{n}$ with edges of lengths $d=\left(d_{1}, \ldots, d_{n}\right)$ and $C$ is independent of $d_{1}, \ldots, d_{n}$, if and only if

$$
\frac{1}{s}=\frac{1}{p}-\frac{1}{q}+\frac{\alpha+\beta}{n}, \quad \alpha<n / p^{\prime}, \quad \beta<n / q .
$$

Proof. Let first (7.9) hold. It is easy to see that all conditions of Theorem 1 are fulfilled and $\mathcal{G} \lesssim 1$. Therefore, Corollary 5 (A) gives (7.8).

On the other hand, (7.8) implies

$$
\sup _{d_{1}, \ldots, d_{n}>0}\left(\prod_{j=1}^{n} d_{j}\right)^{-1 / s}\left\|S_{Q_{d}}\right\|_{L_{\alpha}^{p}\left(\mathbb{R}^{n}\right) \rightarrow L_{-\beta}^{q}\left(\mathbb{R}^{n}\right)} \lesssim 1 .
$$

Then using (7.7) with $r=s^{\prime}$ and $d_{1}=\ldots=d_{n}=z$ we arrive at $z^{n\left(\frac{1}{p}-\frac{1}{q}-\frac{1}{s}\right)+\alpha+\beta} \lesssim \mathcal{K} \lesssim 1$ for any $z>0$. This yields $\frac{1}{s}=\frac{1}{p}-\frac{1}{q}+\frac{\alpha+\beta}{n}$.

Using a method similar to the proof of Corollary 3 , one can verify that conditions $\alpha<n / p^{\prime}$ and $\beta<n / q$ hold. Let us show, for example, that $\beta<n / q$. Indeed, let $C_{\xi}(\bar{z})$ be a cube with the edge length $\xi$ centered at $\bar{z}=\left(z_{1}, \ldots, z_{n}\right)$. Fix $d_{1}, \ldots, d_{n}>0$ and set $d_{0}:=\max _{1 \leqslant i \leqslant n} d_{i}$. Notice that if $x \in C_{\delta}(\overline{0})$, where $\delta<\pi / 4 d_{0}$, and $y \in C_{\pi /\left(4 d_{0}\right)}(\overline{\pi / d})$, then $\frac{\pi}{4} \leqslant \frac{d_{i}\left|x_{i}-y_{i}\right|}{2} \leqslant \frac{3 \pi}{4}, i=1, \ldots, n$. Therefore, 


$$
\begin{aligned}
& \left.\left\|S_{Q_{d}}\right\|_{L^{p}\left(\mu^{-1} ; \mathbb{R}^{n}\right) \rightarrow L^{q}\left(\nu ; \mathbb{R}^{n}\right)} \gtrsim\left|C_{\pi /\left(4 d_{0}\right)}(\overline{\pi / d})\right|^{-\frac{1}{p}}|| y\right|^{-\beta} \int_{C_{\pi /\left(4 d_{0}\right)}(\overline{\pi / d})}|x|^{-\alpha} \prod_{j=1}^{n} \frac{\sin \left(d_{j}\left(y_{j}-x_{j}\right) / 2\right)}{\left(y_{j}-x_{j}\right) / 2} d x \|_{L_{q}\left(\mathbb{R}^{n}\right)} \\
& \geqslant\left|C_{\pi /\left(4 d_{0}\right)}(\overline{\pi / d})\right|^{-\frac{1}{p}}\left(\int_{C_{\delta}(\overline{0})}\left(|y|^{-\beta} \int_{C_{\pi /\left(4 d_{0}\right)}(\overline{\pi / d})}|x|^{-\alpha} \prod_{j=1}^{n} \frac{\sin \left(d_{j}\left(y_{j}-x_{j}\right) / 2\right)}{\left(y_{j}-x_{j}\right) / 2} d x\right)^{q} d y\right)^{1 / q} \\
& \gtrsim\left(\int_{C_{\delta}(\overline{0})}|y|^{-\beta q} d y\right)^{1 / q} .
\end{aligned}
$$

This implies $\beta<n / q$.

\section{References}

[Be] W. Beckner, Pitt's inequality with sharp convolution estimates, Proc. Amer. Math. Soc., 136(5) (2008), 1871-1885.

[BS] C. Bennett, R. Sharpley, Interpolation of Operators, Academic Press, 1988.

[BGT] N. H. Bingham, C. M. Goldie, J. L. Teugels, Regular Variation, Cambridge University Press, 1989.

[Bu1] H.-Q. Bui, Weighted Young's inequality and convolution theorems on weighted Besov spaces, Math. Nachr., 170 (1994), 25-37.

[Bu2] H.-Q. Bui, Weighted Besov and Triebel spaces: Interpolation by the real method, Hiroshima Math. J., 12, 3 (1982), 581-605.

[COV] C. Cascante, J. Ortega, I. Verbitsky, Trace inequalities of Sobolev type in upper triangle, Proc. London Math. Soc., 80 (2000), 391-414.

[DL] R. A. DeVore, G. G. Lorentz, Constructive Approximation, Springer-Verlag, 1993.

[DD] P. L. De Nápoli, I. Drelichman, Weighted convolution inequalities for radial functions, arXiv:1210.1206. 2012.

[DT] Z. Ditzian, S. Tikhonov, Ul'yanov and Nikol'skii-type inequalities, J. Approx. Theory, 133, 1 (2005), 100133.

[Du] J. Duoandikoetxea, Fractional integrals on radial functions with applications to weighted inequalities, Annali Mat. Pura Appl., DOI: 10.1007/s10231-011-0237-7

[GK] M. Gabidzashvili, V. Kokilashvili, Two weight weak type inequalities for fractional type integrals, Ceskoslovenska Akademie Ved., 45 (1989), 1-11.

[Ke] R. A. Kerman, Convolution theorems with weights, Trans. Amer. Math. Soc., 280 (1983), $207-219$.

[LMPT] M. Lacey, K. Moen, C. Pérez, R. H. Torres, Sharp weighted bounds for fractional integral operators, J. Funct. Anal., 259, 5, (2010) 1073-1097.

[MT] G. Mastroianni, V. Totik, Weighted polynomial inequalities with doubling and $A_{\infty}$ weights. Constr. Approx. 16, 1 (2000), 37-71.

[Ma] V. G. Maz'ya, Sobolev Spaces. Springer-Verlag, Berlin, 1985.

[MN] V. G. Maz'ya, Y. Netrusov, Some counterexamples for the theory of Sobolev spaces on bad domains, Potential Anal., 4 (1995), 47-65.

[MV] M. Meyries, M. Veraar, Sharp embedding results for spaces of smooth functions with power weights, Studia Mathematica, 208 (2012), 257-293.

[MW] B. Muckenhoupt, R. Wheeden, Weighted norm inequalities for fractional integrals, Trans. Amer. Math. Soc., 192 (1974), 261-274.

[NT1] E. Nursultanov, S. Tikhonov, Convolution inequalities in Lorentz spaces, J. Fourier Anal. Appl., Vol. 17 (2011), 486-505.

[NT2] E. Nursultanov, S. Tikhonov, Net spaces and boundedness of integral operators. J. Geom. Anal., 21 (2011), 950-981. 
[ON] R. O'Neil, Convolution operators and $L(p, q)$ spaces, Duke Math. J., 30 (1963), 129-142.

[Pe] C. Pérez, Two weighted inequalities for potential and fractional type maximal operators, Indiana Univ. Math. J., 43, 2 (1994), 663-683.

[Sa1] E. Sawyer, A two weight weak type inequality for fractional integrals, Trans. Amer. Math. Soc., 281 (1984), 339-345.

[Sa2] E. Sawyer, A characterization of a two-weight norm inequality for fractional and Poisson integrals, Trans. Amer. Math. Soc. 308, 2 (1988), 533-545.

[SaWh] E. Sawyer, R. Wheeden, Weighted inequlities for fractional integrals on Euclidean and homogeneous spaces, Amer. J. Math, 114 (1992), 813-874.

[So] S. L. Sobolev, Some Applications of Functional Analysis in Mathematical Physics. Providence, RI, 1991.

[SW] E. Stein, G. Weiss, Introduction to Fourier analysis on Euclidean spaces. Princeton, N.J., 1971.

[SW1] E. Stein, G. Weiss, Fractional integrals on n-dimensional Euclidean space. J. Math. Mech. 7 (1958), 503514.

[Tr] H. Triebel, Theory of Function Spaces, Birkhäuser Verlag, Basel, 1983.

E. Nursultanov, Lomonosov Moscow State University (Kazakh Branch) and Gumilyov Eurasian National University, Munatpasova 7, 010010 Astana Kazakhstan

E-mail address: er-nurs@yandex.ru

S. Tikhonov, ICREA and Centre de Recerca Matemàtica (CRM), E-08193, Bellaterra, Barcelona

E-mail address: stikhonov@crm.cat 